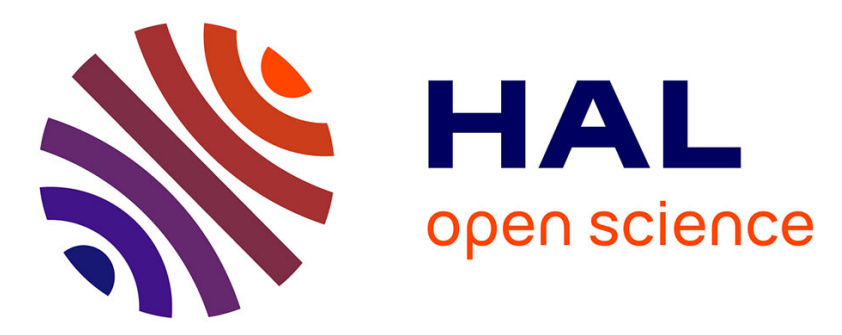

\title{
Stiffness Modeling of Compliant Serial Manipulators Based on Tensegrity Mechanism under External Loading
}

Wanda Zhao, Anatol Pashkevich, Damien Chablat

\section{To cite this version:}

Wanda Zhao, Anatol Pashkevich, Damien Chablat. Stiffness Modeling of Compliant Serial Manipulators Based on Tensegrity Mechanism under External Loading. 18th International Conference on Informatics in Control, Automation and Robotics (ICINCO), Jul 2021, Paris, France. 10.5220/0010506102540262. hal-03311957

\section{HAL Id: hal-03311957 \\ https://hal.science/hal-03311957}

Submitted on 2 Aug 2021

HAL is a multi-disciplinary open access archive for the deposit and dissemination of scientific research documents, whether they are published or not. The documents may come from teaching and research institutions in France or abroad, or from public or private research centers.
L'archive ouverte pluridisciplinaire HAL, est destinée au dépôt et à la diffusion de documents scientifiques de niveau recherche, publiés ou non, émanant des établissements d'enseignement et de recherche français ou étrangers, des laboratoires publics ou privés. 


\title{
Stiffness Modeling of Compliant Serial Manipulators Based on Tensegrity Mechanism under External Loading
}

\author{
Wanda Zhao ${ }^{1}$, Anatol Pashkevich ${ }^{1,2}$, Damien Chablat ${ }^{1,3}$ \\ ${ }^{1}$ Laboratoire des Sciences du Numérique de Nantes (LS2N), UMR CNRS 6004, Nantes, France \\ ${ }^{2}$ IMT Atlantique Bretagne Pays de la Loire, Nantes, France \\ ${ }^{3}$ Centre National de la Recherche Scientifique (CNRS), France \\ Wanda.Zhao@ls2n.fr,Anatol.Pashkevich@imt-atlantique.fr, Damien.Chablat@cnrs.fr.
}

Keywords: Compliant manipulator; Tensegrity mechanism; Stiffness analysis; Robot buckling.

\begin{abstract}
The paper focuses on the stiffness modeling of a new type of compliant manipulator and its non-linear behavior under external loading. The manipulator under study is a serial mechanical structure composed of dual-triangle segments. The main attention is paid to the possible equilibriums and the manipulator stiffness behavior under the loading for the initial non-straight configuration. It was demonstrated that there is a quasi-buckling phenomenon for this manipulator while the external loading increasing. In the neighborhood of these configurations, the manipulator behavior was analyzed using the enhanced Virtual Joint Method (VJM). Relevant simulation study confirmed the obtained theoretical results.
\end{abstract}

\section{INTRODUCTION}

Compliant manipulators are used nowadays in many fields due to their flexibility, modularized construction, and low weight. A lot of new mechanical structures were studied in this area (Frecker, Ananthasuresh et al., 1997), (AlbuSchaffer et al., 2008), (Wang and Chen, 2009), (Howell, 2013), which showed quite good performances compared with traditional rigid robots. Recently, in literature particular attention is paid to tensegrity mechanisms, which are made up of a series of similar segments composed of compressive and tensile elements (cables or springs) (Skelton and Oliveira, 2009), (Moored, Kemp, et al., 2011). One of such structures is studied in this paper.

Stiffness properties of some tensegrity mechanisms have been already studied carefully. In (Arsenault and Gosselin, 2006), the authors considered the mechanism composed of two springs and two length-changeable bars. They analyzed the mechanism stiffness using the energy method, demonstrated that the mechanism stiffness may decrease under external loading with the actuators locked, which may lead to the "buckling" phenomenon. Also, in (Furet, Lettl and Wenger, 2018), the cable-driven X-shape tensegrity structures were considered; here the authors investigated the influence of cable lengths on the mechanism equilibrium configurations, which may be both stable and unstable. The relevant analysis of the equilibrium configurations as well as the stability and singularity study can be found in (Wenger and Chablat, 2019).

For robotics, similar to classical mechanics dealing with the Euler column, the buckling is usually treated as an undesirable phenomenon, because the robot may suddenly change its shape when the loading force exceeds some critical value. However, such property can be useful in some fields (Yamada, Mameda, et. al., 2010). Also, sometimes the quasi-buckling phenomenon may occur, which changes the robot resistance in one direction suddenly while the external loading is increasing. It is not typical for robotics and was rarely studied before. For this reason, this phenomenon should be obligatory taken into account in stiffness analysis.

This paper is an extension of our previous results (Zhao, Pashkevich et al., 2020 \& 2021), which concentrated on the stiffness analysis of the simplest manipulator composed of two and three segments. It was assumed that each segment is a composition of two rigid triangle parts, which are connected by a passive joint in the center and two elastic edges on each side with controllable preload. In contrast to the previous results, here we consider a general case 

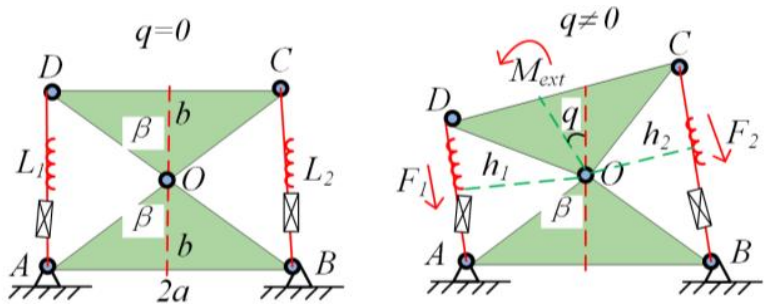

Figure 1: Geometry of a dual-triangle mechanism (a) monotonic case

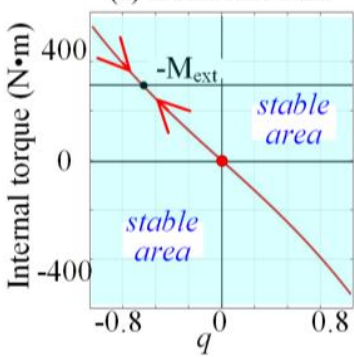

(b) non-monotonic case

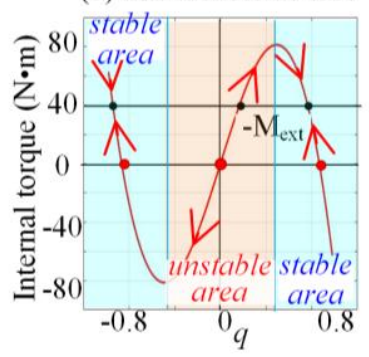

Figure 2: The torque-angle curves of dual-triangle mechanism

with an arbitrary number of segments, and its stiffness behavior under the loading.

\section{MECHANICS OF A SINGLE SEGMENT}

Let us present first a single segment of the compliant serial manipulator under study. It consists of two rigid triangles connected by a passive joint whose rotation is constrained by two linear springs as shown in Fig. 1. It is assumed that the mechanism geometry is described by two triangle parameters ( $a$, $b)$, and the mechanism shape is defined by the central angle $q$, which is adjusted through two control inputs influencing on the springs $L_{1}$ and $L_{2}$. Let us denote the spring lengths in the non-stress state as $L^{0}$, and the spring stiffness coefficient as $k$.

The mechanism configuration angle $q$ corresponding to the given control inputs $L^{0}$ can be computed through the static equilibrium equation of this mechanism, which can be easily derived using the forces generated by the springs: $F_{i}=k_{i}\left(L_{i}-L_{i}^{0}\right)$, where the lengths $L_{i}$ are computed using the formulas $L_{i}(q)=c \sqrt{2+2 \cos \left(\theta_{i}\right)}, \quad c=\sqrt{a^{2}+b^{2}}$, $\theta_{1}=2 \beta+q, \theta_{2}=2 \beta-q$, and $\beta=\operatorname{atan}(a / b)$. It can be proved that the torques generated by the springs can be obtained as the following form.

$$
\begin{aligned}
& M_{1}(q)=+k\left(1-L^{0} / L_{1}(q)\right) c^{2} \sin (2 \beta+q) \\
& M_{2}(q)=-k\left(1-L^{0} / L_{2}(q)\right) c^{2} \sin (2 \beta-q)
\end{aligned}
$$

where $k$ denote the springs stiffness coefficients, $L_{1}(q)$ and $L_{2}(q)$ are the spring lengths, $L^{0}$ are control inputs, while $c$ and $\beta$ are the geometric parameters described above (see Fig. 1). So, taking into account the external torque $M_{\text {ext }}$ applied to the moving platform, the static equilibrium equation for the considered mechanism can be written as $M(q)+M_{\text {ext }}$ $=0$, where $M(q)=M_{1}(q)+M_{2}(q)$ and

$M(q)=2 c k\left[c \cos (2 \beta) \sin q-L^{0} \cos (\beta) \sin (q / 2)\right]$

It should be noted that the static stability of this mechanism highly depends on the equilibrium configuration defined by $q$. As follows from the relevant analysis, the function $M(q)$ can be either a monotonic or non-monotonic one (Fig. 2), so the single-segment mechanism may have multiple stable and unstable equilibriums, which are studied in detail in (Zhao, Pashkevich et al. 2020). As follows from the relevant analysis, the stability condition for this mechanism can be expressed via the derivative sign at the zero point, i.e. $\left.M^{\prime}(q)\right|_{q=0}<0$, which is easy to verify in practice. So, the relevant analytical expression for the derivative

$M^{\prime}(q)=c k\left[2 c \cos (2 \beta) \cos q-L^{0} \cos \beta \cos (q / 2)\right]$

allows us to present the condition of the torqueangle curve monotonicity as follows

$$
L^{0}>2 b \cdot\left(1-(a / b)^{2}\right)
$$

This expression is extensively used below.

\section{MECHANICS OF MULTI- SEGMENT MANIPULATOR}

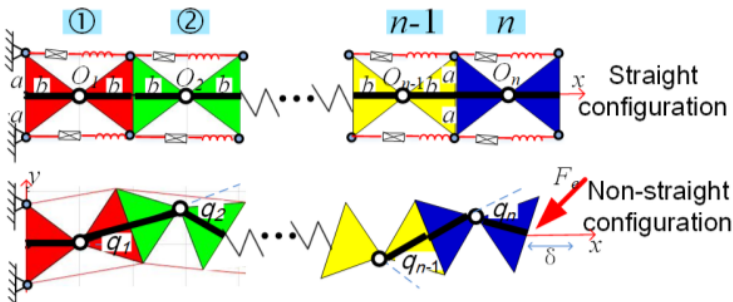

Figure 3: Geometry of a multi-segment manipulator. 
Table 1 Two typical initial configurations of the manipulator for the end-point location $(\mathrm{x} 0, \mathrm{y} 0)=(7.7 \mathrm{~b}, 0)$.

\begin{tabular}{|l|ll|c|c|c|c|}
\hline & \multicolumn{2}{|c|}{ Initial shape } & \multicolumn{4}{c|}{ Initial configuration angles } \\
\cline { 3 - 7 } & & $q 1$ & $q 2$ & $q 3$ & $q 4$ \\
\hline Case \#1 & U-shape: & -0.3093 & +0.1348 & +0.4246 & +0.2288 \\
\hline Case \#2 & Z-shape: & Ya & -0.1136 & +0.3768 & -0.6242 & +0.7869 \\
\hline
\end{tabular}

The serial manipulator considered in this paper is composed of $n$ similar sections connected in series as shown in Fig. 3, where the left-hand-side is assumed to be fixed. For the initial straight configuration, the stiffness properties of this manipulator were studied in our previous paper, where the buckling phenomenon (similar to the Euler column) was discovered and the critical force was computed. In this paper, a general case is considered where the initial shape is assumed to be non-straight, and the stiffness analysis is carried out for the loaded mode.

Let us assumed that the initial configuration of the $n$-link manipulator is a non-straight one, which corresponds to the non-zero angles ( $\left.q_{i}^{0} \neq 0, i=1,2, \ldots, n\right)$ and the initial end-point location is $\left(x_{0}, y_{0}\right)=(2 n \cdot b-\Delta x, 0)$ with $\Delta x>0$. It is assumed that the corresponding control inputs $\left(L_{i 1}^{0}, L_{i 2}^{0}\right) i=1,2, . ., n$ are computed from the equilibrium conditions, where $L_{i 1}^{0}=L^{0}-\Delta_{i}$, $L_{i 2}^{0}=L^{0}+\Delta_{i}$ and $L^{0}=b$ (causing the pre-stress). It is clear that if $n \geq 3$ this manipulator is redundant with respect to the end-effector location control in the $(x$, $y$ )-plane. So, for given $\left(x_{0}, y_{0}\right)$ the configuration angles $q_{i}^{0}$ cannot be computed in a unique way. For this reason, we will consider two typical initial shapes of the manipulator, which in our previous paper were referred to as the U-shape and Z-shape (Zhao, Pashkevich et al. 2020). Examples of such initial configurations for $n=4$ are shown in Table 1 , and their elastostatic properties will be carefully studied below.

First, let us investigate the force-deflection relations $F_{x}(\delta x)$ and $F_{y}(\delta x)$ corresponding to the end-effector displacement with $\delta y=0$, i.e. from the initial location $\left(x_{0}, y_{0}\right)=(2 n \cdot b-\Delta x, 0)$ to the current one $(x, y)=(2 n \cdot b-\Delta x-\delta x, 0)$ where $\delta x$ is the end-effector deflection caused by the external forces $\left(F_{x}, F_{y}\right)$ and $\Delta x$ denotes the initial displacement of the end-effector. Let us apply the energy method (detailed of this elastic energy were in Zhao, 2020) allowing us to find possible equilibrium configurations corresponding to the given $\delta x$. It should be noted that the geometric constraint coming from the given end-effector location is

$$
\begin{array}{r}
b+2 b \sum_{j=1}^{n-1}\left(\cos \sum_{i=1}^{j} q_{i}\right)+b \cos \sum_{i=1}^{n} q_{i}=2 n b-\Delta x-\delta_{x} \\
2 b \sum_{j=1}^{n-1}\left(\sin \sum_{i=1}^{j} q_{i}\right)+b \sin \sum_{i=1}^{n} q_{i}=0
\end{array}
$$

and allows us to reduce the number of variables in the energy function $E\left(q_{1}, q_{2}, \ldots q_{n-2}\right)$ by applying the 2-link manipulator inverse kinematics to compute the remaining angles $\left(q_{n-1}, q_{n}\right)$. Further, by detecting the $\mathrm{max} / \mathrm{min}$ and saddle points of the function $E\left(q_{1}, q_{2}, \ldots q_{n-2}\right)$, it is possible to find the

\section{Energy $E\left(q_{1}, q_{2}\right)$ for initial U-shape, $\delta x / b=0.4$}
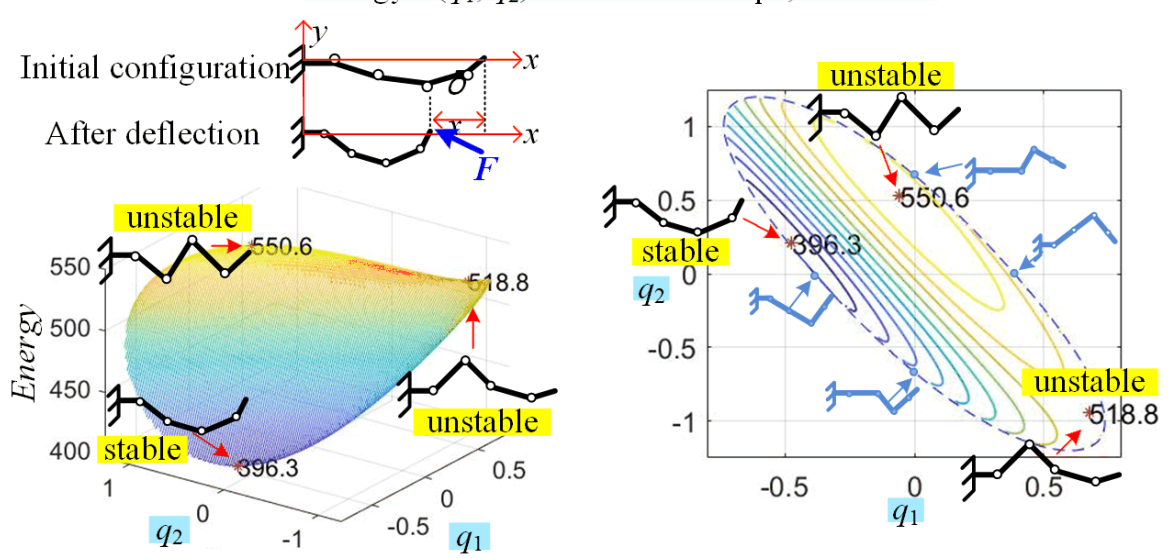

Figure 4: The energy function $E\left(q_{1}, q_{2}\right)$ and manipulator equilibriums for initial U-shape configuration (end-effector deflection $\delta x / b=0.4, \delta y=0$; geometric parameters $a / b=1.0 ; q 4>0$ ). 


\section{Energy $E\left(q_{1}, q_{2}\right)$ for initial Z-shape, $\delta x / b=0.2$}

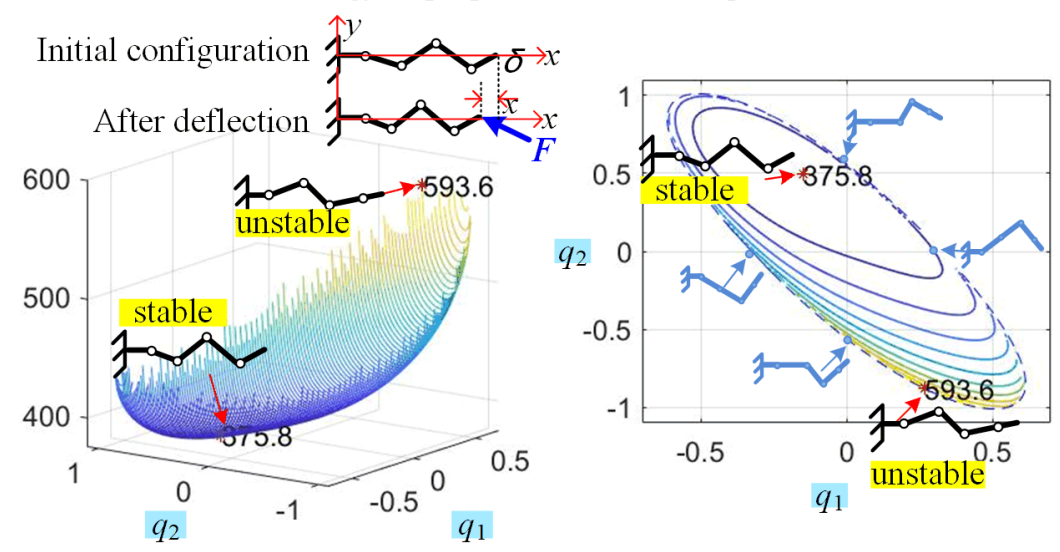

Figure 5: The energy function $E\left(q_{1}, q_{2}\right)$ and manipulator equilibriums for initial Z-shape configuration (end-effector deflection $\delta x / b=0.2, \delta y=0$; geometric parameters $a / b=1.0 ; q 4>0$ ).

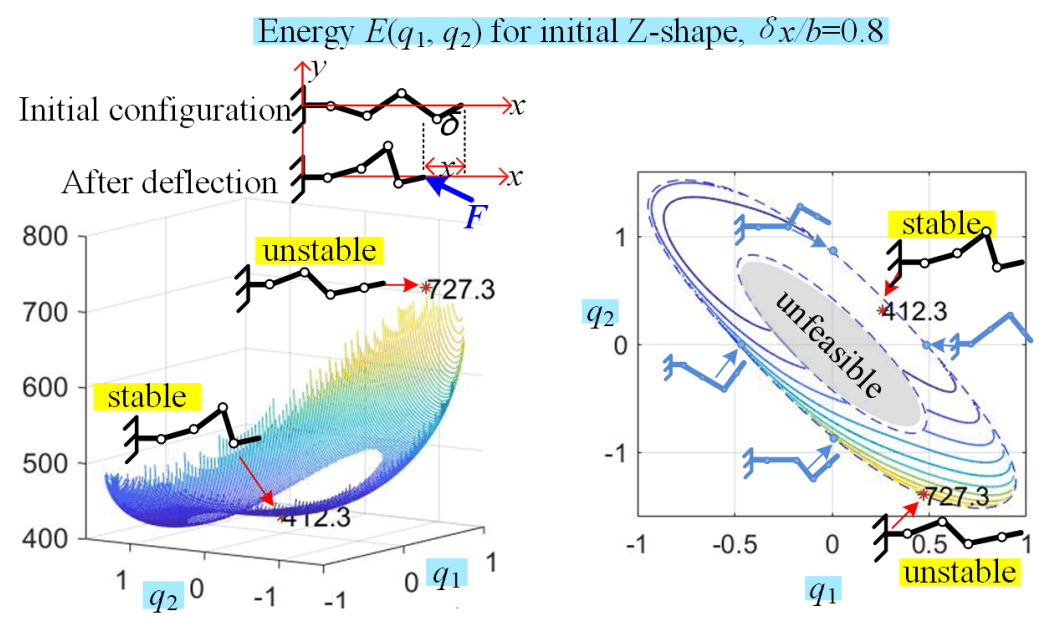

Figure 6:The energy function $E\left(q_{1}, q_{2}\right)$ and manipulator equilibriums for initial U-shape configuration (end-effector deflection $\delta x / b=0.8, \delta y=0$; geometric parameters $a / b=1.0 ; q 4>0$ ).

configuration angles for all possible equilibriums To evaluate their stability and compute the external forces $\left(F_{x}, F_{y}\right)$ corresponding to the end-effector deflection $\delta x$, let us apply the Moore-Penrose pseudo-inverse on the static equilibrium condition, which is shown as follows,

$$
\left[\begin{array}{l}
F_{x} \\
F_{y}
\end{array}\right]=-\left[\mathbf{J}_{\mathbf{q}}^{\mathrm{T}}\right] \cdot\left[\mathbf{J}_{\mathbf{q}} \mathbf{J}_{\mathbf{q}}^{\mathrm{T}}\right]^{-1} \cdot\left[\begin{array}{c}
M_{q 1} \\
\ldots \\
M_{q n}
\end{array}\right]
$$

where both the Jacobian $\mathbf{J}_{\mathbf{q}}$ and the joint torques $M q_{i}$ are computed using the configuration angles $q_{i}$ corresponding to the stable equilibriums.

Examples of the obtained energy surfaces for $n=4$ are presented in Figs 4, 5 and 6, where the endeffector elastic deflection is $\delta x \in\{0.2 b, 0.4 b, 0.8 b\}$ and the initial shapes correspond to the end-effector displacement $\Delta x=0.3 b$ (see Table 1). As follows from these figures, for the initial U-shape (see Fig. 4) there are two cases of the energy surfaces $E\left(q_{1}, q_{2}\right)$ corresponding to $q_{4}>0$ and $q_{4}<0$ which are symmetrical. Totally, they have 6 critical points; each of them contains a single maximum, a single minimum and a single saddle point. Also, their evolution with respect to $\delta x$ is continuous, their (申opology remains the same while increasing the deflection $\delta x$. In contrast, for the initial Z-shape (see Figs. 5, 6), the energy surfaces $E\left(q_{1}, q_{2}\right)$ are quite different, their evolution with respect to $\delta x$ is discontinuous. The latter leads to sign-changing of some configuration angles $q_{i}$ under the external loading $F$ as shown in the figures (see angle $q_{1}$ for instance). Besides, if the deflection $\delta x$ is large enough as in Fig. 6, the energy surfaces may contain a "hole", i.e. an unfeasible area, caused by the 
Initial U-shape, case \#1

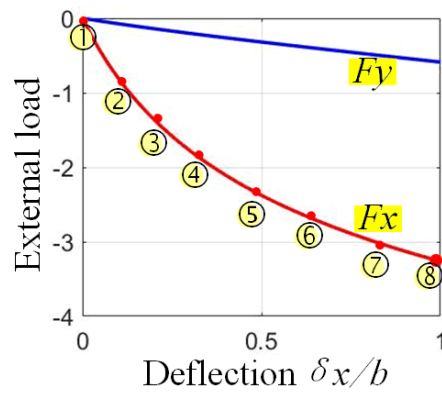

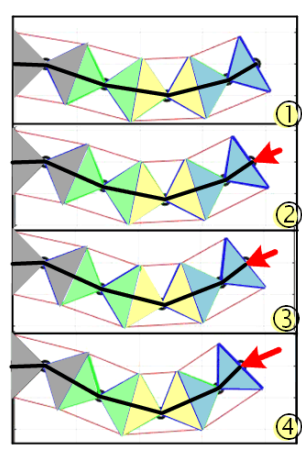

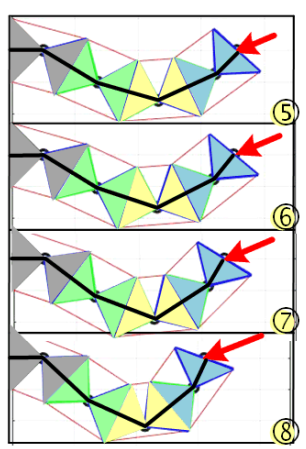

Figure 7: Force-deflection curves $F x(\delta x), F y(\delta x)$ and manipulator shape changing under the loading for initial U-shape for $(x 0, y 0)=(7.7 \mathrm{~b}, 0)$, geometric parameters $a / b=1.0$ and $\delta y=0$.

Initial Z-shape, case \#2

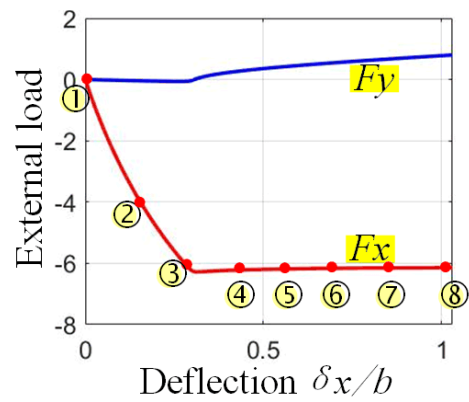

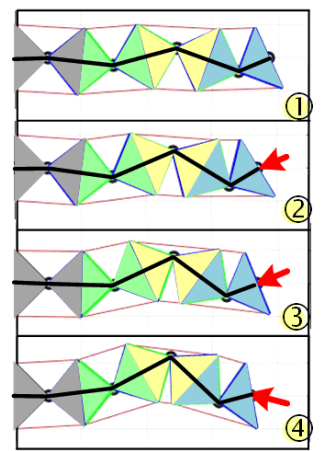

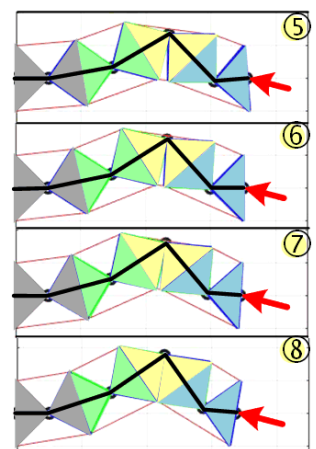

Figure 8: Force-deflection curves $F_{x}(\delta x), F_{y}(\delta x)$ and manipulator shape changing under the loading for initial Z-shape for $\left(x_{0}, y_{0}\right)=(7.7 b, 0)$, geometric parameters $a / b=1.0$ and $\delta y=0$.

violation of the geometric constraints $\left|q_{i}\right| \leq q_{i}^{\max }$ inside of the manipulator segments.

\section{MANIPULATOR STIFFNESS UNDER THE LOADING}

By applying the above-presented energy method and computing minimums of the energy function $E\left(q_{1}, q_{2}, \ldots q_{n-2}\right) \rightarrow \min$ for different $\delta x$, it is possible to obtain the desired force-deflection relations $F_{x}(\delta x)$ and $F_{y}(\delta x)$ describing the manipulator stiffness properties. Examples of such computations for $n=4$ are presented in Figs 7 and 8 .

For the initial U-configuration (see Fig. 7), the change of the manipulator shape is smooth, the manipulator resistance against the external loading is gradually increasing while the deflection $\delta x$ becomes larger. Also, the stiffness coefficient in the $x$-direction is decreasing continuously. This tendency is observed until the manipulator reaches its geometric constraints.

In contrast, for the initial Z-configuration (see Fig. 8), there are two intervals of the manipulator deformation. In the beginning when $\delta x$ is relatively small the manipulator maintains its Z-shape and the resistance against the external force is monotonically increasing, similar to the previous case. Further, when the deflection $\delta x$ is larger than some critical value, the buckling phenomenon is occurring, and the manipulator resistance against the external force is not increasing anymore. Correspondingly, the stiffness coefficient $d F_{x} / d x$ becomes very small, the stiffness coefficient $d F_{y} / d x$ changes its sign and the manipulator does not keep its initial Z-shape (some of the angles $q_{i}$ change the signs). Finally, after the buckling, the manipulator moves in the direction of its internal geometric constraints. Hence, in practice, it is preferable to use the U-shape of the manipulator if the task space obstacles (external constraints) allows. It should be also noted that for the Z-shape it is necessary to avoid high loadings exceeding the critical force causing buckling.

Further, in addition to the above presented forcedeflection relations $F_{x}(\delta x)$ and $F_{y}(\delta x)$ derived from the assumption of $\delta x=$ var , $\delta y=0$, let us analyze the changing of the manipulator stiffness coefficients under the loading $\left(F_{x}, F_{y}\right)$ without imposing any kinematic constraints of the end- 
effector location. To obtain the desired relations it is necessary to compute the configuration angles $\left(q_{1}, \ldots, q_{n}\right)$ corresponding to the manipulator equilibriums for different given external forces $\left(F_{x}, F_{y}\right)$. It is clear that these angles can be found numerically by solving the system of $n$ independent equations

$$
\mathbf{M}_{\mathbf{q}}+\left[\mathbf{J}_{\mathbf{q}}^{\mathrm{T}}\right]_{\mathbf{n} \times \mathbf{2}} \mathbf{F}=\mathbf{0}
$$

describing the static equilibrium condition (by applying Newton's method for instance). However, the initial guess of the angles $\left(q_{1}^{0}, \ldots, q_{n}^{0}\right)$ should be evaluated correctly, to ensure that they are in the neighborhood of the minimum energy configuration, because only such cases can be observed in practice. Such initial guess can be obtained using the abovepresented energy method applied in the space $\left(q_{1}, \ldots, q_{n}\right)$ with rather rough grid with large step. Also, the desired angles corresponding to the external loading $\left(F_{x}, F_{y}\right)$ can be found using the Matlab function fminsearch which minimizes the sum of the squared residuals i.e.

$$
\mathbf{q}=\arg \min _{\mathbf{q}}\left(\left\|\left[\mathbf{M}_{\mathbf{q}}\right]_{n \times 1}+\left[\mathbf{J}_{\mathbf{q}}^{\mathrm{T}}\right]_{n \times 2} \cdot \mathbf{F}\right\|^{2}\right)
$$

where both the internal torques $\mathbf{M}_{\mathbf{q}}$ and the Jacobian $\mathbf{J}_{\mathbf{q}}$ depend on the angles $\left(q_{1}, \ldots, q_{n}\right)$. It should be also mentioned that it is possible to simplify the problem of the initial guess $\left(q_{1}^{0}, \ldots, q_{n}^{0}\right)$ selection by gradually increasing the forces $\left(F_{x}, F_{y}\right)$ and using solutions from the previous loadedequilibrium as the initial guess for the next one corresponding to $\left(F_{x}+\Delta F_{x}, F_{y}+\Delta F_{y}\right)$. However, when the forces $\left(F_{x}, F_{y}\right)$ approach the buckling point, the initial guess from the previous step is not suitable because the configuration angles are changing essentially and only the straightforward energy method allows to obtain the correct initial guess.

\section{EVOLUTION OF STIFFNESS COEFFICIENTS}

If the equilibrium configuration angles $\left(q_{1}, \ldots, q_{n}\right)$ corresponding to the given force $\left(F_{x}, F_{y}\right)$ are computed, it is possible to find the desired stiffness coefficients using the formula for the loaded case,

$$
\mathbf{K}_{\mathbf{F}}=\left[\mathbf{J}_{\mathbf{q}}\left(\mathbf{K}_{\mathbf{q}}-\mathbf{K}_{\mathbf{g}}\right)^{-1} \mathbf{J}_{\mathbf{q}}^{\mathrm{T}}\right]^{-1}
$$

that includes two essential components, the first of which $\mathbf{K}_{\mathbf{q}}$ corresponds to the unloaded case, and the second one $\mathbf{K}_{\mathrm{g}}$ describes the external force influence on the stiffness. In this expression, the $n \times n$ matrix of the joint elastic stiffness coefficients $\mathbf{K}_{\mathbf{q}}=\operatorname{diag}\left(K_{e q 1}, \ldots, K_{e q i}\right)$ can be computed using the segment torque equilibrium equation from section 2 , which yields

$$
\begin{aligned}
& K_{e q i}=2 k\left(b^{2}-a^{2}\right) \cos q_{i}- \\
& -k\left(\frac{L_{i 1}^{0}+L_{i 2}^{0}}{2} b \cos \frac{q_{i}}{2}-\frac{L_{i 1}^{0}-L_{i 2}^{0}}{2} a \sin \frac{q_{i}}{2}\right)
\end{aligned}
$$

It should be stressed that here, the control inputs $L_{i 1}^{0}$ and $L_{i 2}^{0}$ are constant values, which correspond to the initial unloaded joint angles $q_{i}$.

The second matrix $\mathbf{K}_{\mathbf{g}}$ containing the stiffness coefficients caused by the loading is symmetrical and can be computed as $\mathbf{K}_{\mathbf{g}} @ \partial \mathbf{J}^{\mathrm{T}} / \partial q_{i} \cdot \mathbf{F}$, which gives us the following formula

$$
\mathbf{K}_{\mathrm{g}}=\left[\begin{array}{c:c:c}
-J_{21} F_{x}+J_{11} F_{y} & \ldots & -J_{2 n} F_{x}+J_{1 n} F_{y} \\
\ldots & \ldots & -J_{2 n} F_{x}+J_{1 n} F_{y}
\end{array}\right]
$$

where $J_{i j}$ denotes the element of the Jacobian matrix $\mathbf{J}_{\mathbf{q}}$ with the $i$ th row and $j$ th colomn.

It is obvious that when the external forces are (\&qual to zero, the stiffness matrix expression is reduced to the form, which is known from the unloaded mode analysis $\mathbf{K}_{0}=\left[\mathbf{J}_{\mathbf{q}} \mathbf{K}_{\mathbf{q}}^{-1} \mathbf{J}_{\mathbf{q}}^{\mathrm{T}}\right]^{-1}$. It should be also mentioned that, in contrast to the classical $n$ link serial manipulators, here the diagonal matrix $\mathbf{K}_{\mathbf{q}}$ is configuration dependent (not constant) because each initial configuration with the angles $\left(q_{1}, \ldots, q_{n}\right)$ produces its own control inputs $L_{i 1}^{0}$ and $L_{i 2}^{0}$ included in the expression (10). Besides, here the unloaded compliance matrix $\mathbf{C}_{0}$ can be expressed analytically in the following way

$$
\mathbf{C}_{0}=\left[\begin{array}{ccc}
\frac{J_{11}^{2}}{K_{q 1}}+\ldots+\frac{J_{1 n}^{2}}{K_{q n}} & * \\
& * & \frac{J_{21}^{2}}{K_{q 1}}+\ldots+\frac{J_{2 n}^{2}}{K_{q n}}
\end{array}\right]
$$

To illustrate the practical importance of the above-presented results, they were applied to the case $n=4$ assuming that the initial (unloaded) endpoint location is $\left(x_{0}, y_{0}\right)=(7.7 \mathrm{~b}, 0)$, and the initial shape is either U- or Z- one. The configuration angles under the loading, corresponding to the external force $F=\left(F_{x}, F_{y}\right)$, were computed numerically using the technique proposed above. Relevant results of the initial Ushape and Z-shape are presented in Figs. 9 and 10 (9tspectively. As follows from these figures, the manipulator stiffness essentially changes if the external loading is applied. For the initial U-shape case, the absolute value of the manipulator stiffness coefficient $\left|K_{\mathrm{xx}}\right|$ decreases first, while the force $F_{\mathrm{x}}$ is 
(a) $F x=v a r, F y=0$

Force-deflection
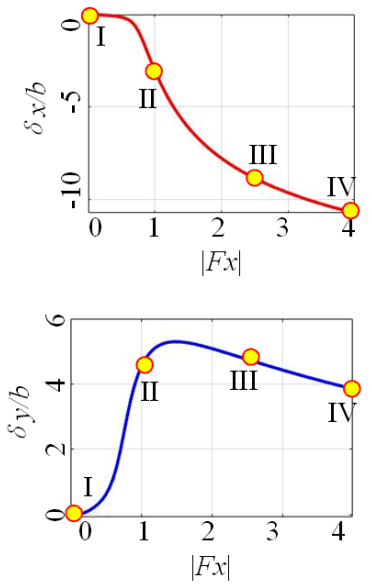

Force-deflection
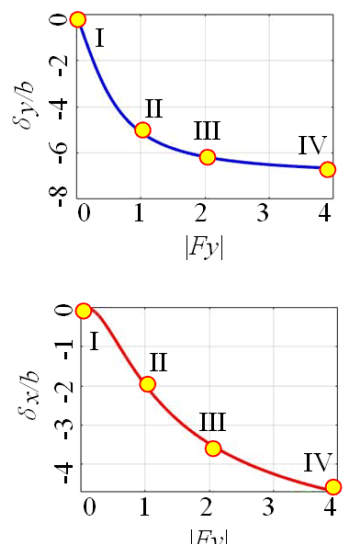

Stiffness coeff.
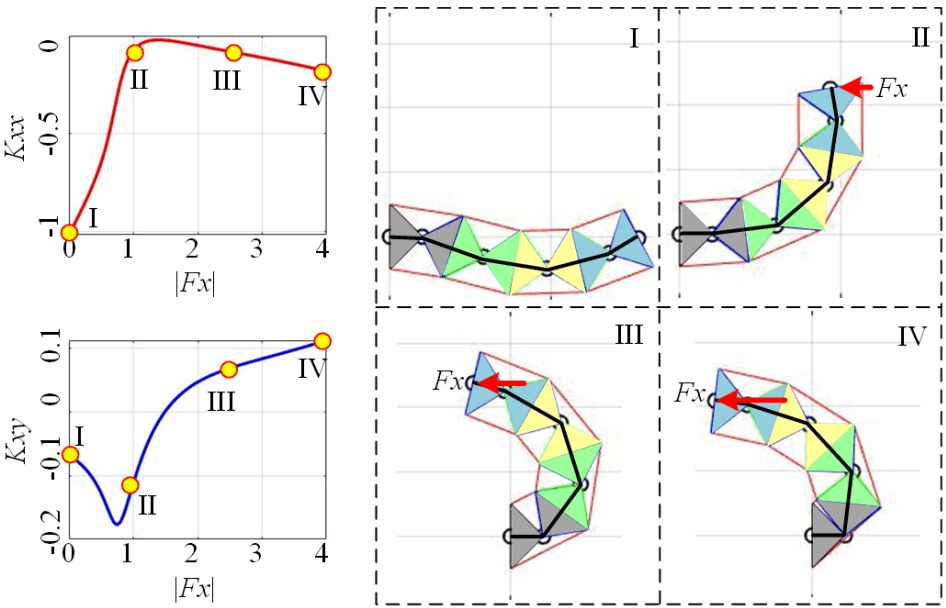

(b) $F x=0, F y=v a r$

Stiffness coeff
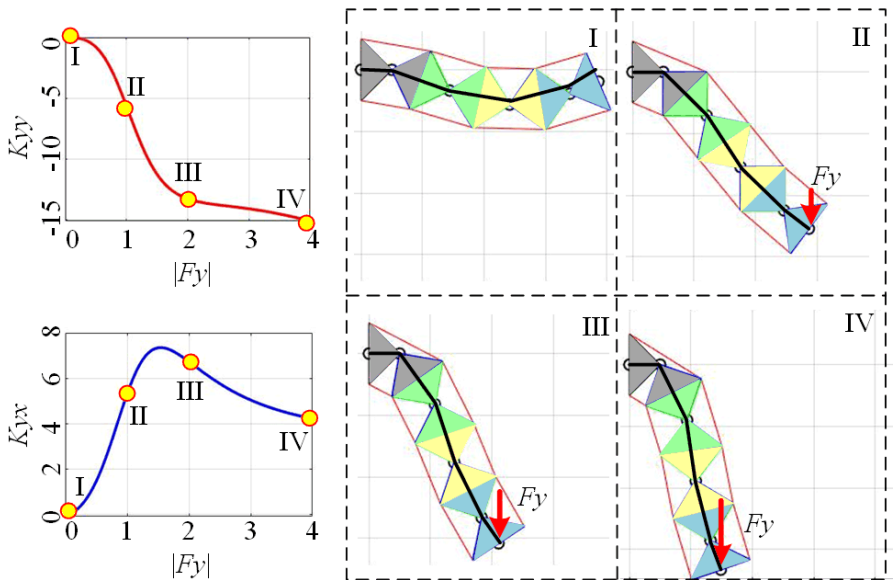

Figure 9: Stiffness coefficients under the $F_{x}$-and $F_{y}$-loading for initial U-shape configuration with $\left(x_{0}, y_{0}\right)=(7.7 b, 0)$ and geometric parameters $a / b=1.0$

increasing (see Fig. 9a), until $F_{\mathrm{x}}$ is reaching some critical value when $\left|K_{\mathrm{xx}}\right|$ is the minimum, then it begins to increase slowly. In contrast, the stiffness coefficient $K_{\mathrm{xy}}$ (describing the manipulator reaction in the $y$-direction) changes its sign under the loading. These stiffness properties can be also interpreted from the geometrical and physical point of view, using the right-hand side of the Fig. 9a, which shows the evolution of the manipulator configuration under the loading. In general, such manipulator behavior can be treated as "quasi-buckling", because for certain loading $F_{\mathrm{x}}$ the stiffness in both $x$ - and $y$ direction is very small. And the manipulator rotates quickly until one of the segment goes close to its joint limits, where the equivalent rotational stiffness coefficient is very low. Hence, in practice, it is necessary to avoid applying too high loading in $x$ - direction causing approaching either to the "quasibuckling" or the joint limits and losing the manipulator stiffness.

On the other side, while increasing the force $F_{\mathrm{y}}$ (i.e. in the orthogonal direction), the absolute value of the stiffness coefficient $\left|K_{\mathrm{yy}}\right|$ is monotonically increasing first, then it keeps the same tendency slowly (see Fig. 9b) because of the restriction of the geometric length of the manipulator. At the same time, the stiffness coefficient $K_{\mathrm{yx}}$ demonstrates nonmonotonic behavior. Such performance can be seen from the evolution of the manipulator configuration at the right-hand side of Fig. 9b, where the manipulator end-point moves towards the extreme location, as far as possible from the initial one. Therefore, the high loading in $y$-direction should be also avoided, to prevent from the manipulator 

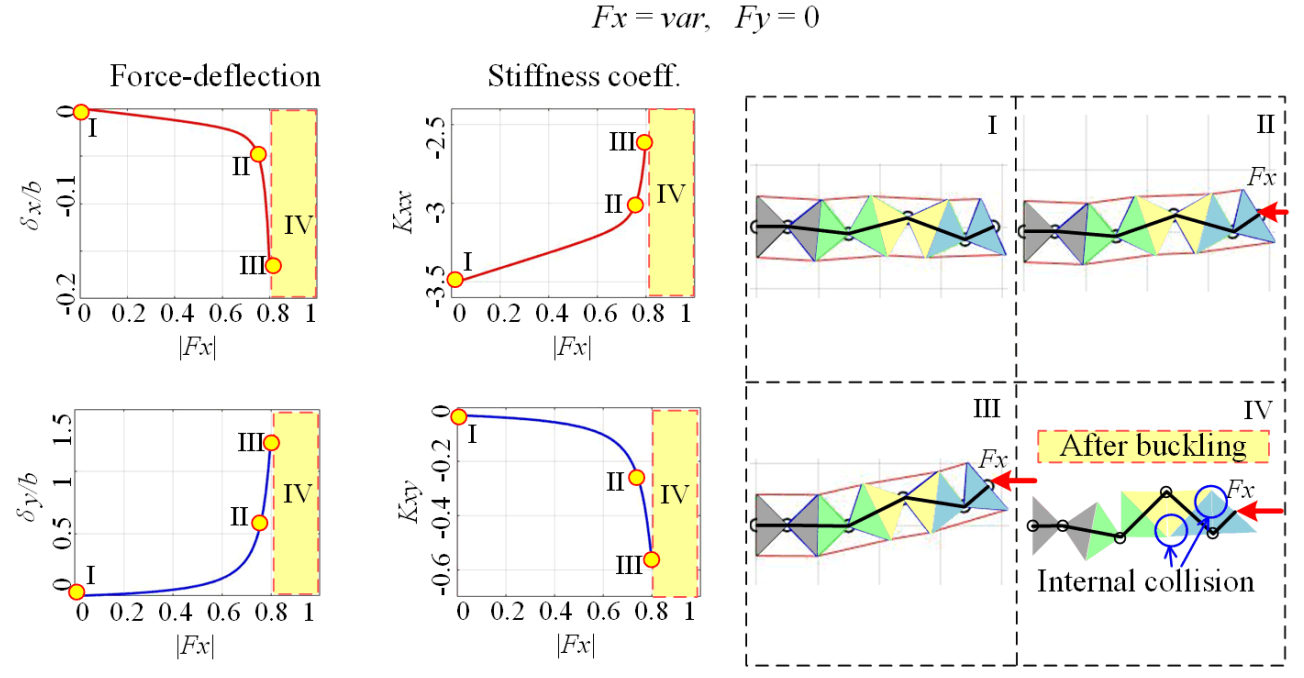

Figure 10: Stiffness coefficients under the $F_{x}$-loading for initial Z-shape configuration with $\left(x_{0}, y_{0}\right)=(7.7 b, 0)$ and geometric parameters $a / b=1.0$

changing its shape change to a pure straight line (see case IV).

However, for the second case study dealing with the initial Z-shape, the stiffness properties under the loading are quite different compared to the U-shape case. In particular, as follows from Fig. 10, under the $F_{\mathrm{x}}$-loading, the absolute value of the stiffness coefficient $\left|K_{\mathrm{xx}}\right|$ decreases gradually at the beginning, then it decrease quickly to zero. In contrast, the absolute value of the stiffness coefficient $\left|K_{\mathrm{xy}}\right|$ increases monotonically. This phenomenon can be also treated as "quasi-buckling" because for certain loading the manipulator stiffness in $x$-direction is equal to zero, and the stiffness in $y$ direction is very high. These results are illustrated geometrically by the right-hand side of Fig. 10 showing the evolution of the manipulator configuration under the $F_{\mathrm{x}}$-loading. It is clear that here each segment of the manipulator tends to move close to its geometric limits before the "quasibuckling" is occurring. In this configuration, even a quite small change of the external force may lead to large manipulator deflection, so in practice, it is reasonable to avoid such situations. It is worth mentioning that the case of $F_{\mathrm{y}}$-loading is not presented in Fig. 10, because it is quite similar to the U-shape case.

Hence, for the manipulator under study, the stiffness properties are essentially non-linear with respect to the loading force. Moreover, if the loading exceeds a certain value, the stiffness coefficients may become very low or even change their sign. The latter may be treated as the quasi-buckling, which normally should be avoided.

\section{CONCLUSIONS}

The paper focuses on the stiffness analysis of a new type of compliant serial manipulator under the loading, which is composed of multiple dual-triangle segments. It is a specific case of the tensegrity mechanisms that currently are widely used in soft robotics. The main attention is paid to the initial non-straight configuration of the manipulator. It was proved that under the external loading there may be the quasi-buckling phenomenon, which suddenly changes the manipulator resistance in one direction of its deflection, but may do not influence the resistance in another direction. It was also demonstrated that normally there are six equilibrium configurations of this manipulator (two stable ones and four unstable ones). But if the deflection of the end-effector is large enough some of the equilibriums may be unfeasible due to the geometric constraints.

To find the possible equilibriums and to analyze the manipulator shape under the loading, the energy method was used. Further, the stiffness analysis was based on the VJM approach allowing to find linearized relations between the end-effector deflection and the external force. Relevant simulation confirmed the obtained results. In the future, this technique will be used for the development of relevant control algorithms and related redundancy resolution. 


\section{ACKNOWLEDGEMENTS}

This work was supported by the China Scholarship Council ( No. 201801810036 ).

\section{REFERENCES}

Frecker, M. I., Ananthasuresh, G. K., Nishiwaki, S., Kikuchi, N., \& Kota, S. (1997). Topological Synthesis of Compliant Mechanisms Using Multi-Criteria Optimization. Journal of Mechanical Design, 119(2), 238-245. https://doi.org/10.1115/1.2826242

Albu-Schaffer, A., Eiberger, O., Grebenstein, M., Haddadin, S., Ott, C., Wimbock, T., Wolf, S., \& Hirzinger, G. (2008). Soft robotics. IEEE Robotics Automation Magazine, 15(3), 20-30. https://doi.org/10.1109/MRA.2008.927979

Wang, M. Y., \& Chen, S. (2009). Compliant Mechanism Optimization: Analysis and Design with Intrinsic Characteristic Stiffness. Mechanics Based Design of Structures and Machines, 37(2), 183-200. https://doi.org/10.1080/15397730902761932

Howell, L. L. (2013). Compliant Mechanisms. In J. M. McCarthy (Ed.), 21st Century Kinematics (pp. 189216). Springer. https://doi.org/10.1007/978-1-44714510-3 7

Skelton, R. E., \& Oliveira, M. C. de. (2009). Tensegrity systems. Springer.

Moored, K. W., Kemp, T. H., Houle, N. E., \& Bart-Smith, H. (2011). Analytical predictions, optimization, and design of a tensegrity-based artificial pectoral fin. International Journal of Solids and Structures, 48(2223), $3142-3159$. https://doi.org/10.1016/i.ijsolstr.2011.07.008

Arsenault, M., \& Gosselin, C. M. (2006). Kinematic, static and dynamic analysis of a planar 2-DOF tensegrity mechanism. Mechanism and Machine Theory, 41(9), 1072-1089. https://doi.org/10.1016/j.mechmachtheory.2005.10.01 $\underline{4}$

Furet, M., Lettl, M., \& Wenger, P. (2019). Kinematic Analysis of Planar Tensegrity 2-X Manipulators. In J. Lenarcic \& V. Parenti-Castelli (Eds.), Advances in Robot Kinematics 2018 (Vol. 8, pp. 153-160). Springer International Publishing. https://doi.org/10.1007/978-3-319-93188-3 18.

Wenger, P., \& Chablat, D. (2019). Kinetostatic analysis and solution classification of a class of planar tensegrity mechanisms. Robotica, 37(7), 1214-1224. https://doi.org/10.1017/S026357471800070X

Yamada, A., Mameda, H., Mochiyama, H., \& Fujimoto, H. (2010). A compact jumping robot utilizing snapthrough buckling with bend and twist. 2010 IEEE/RSJ International Conference on Intelligent Robots and Systems, 389-394. https://doi.org/10.1109/IROS.2010.5652928

Zhao, W.; Pashkevich, A.; Klimchik, A. and Chablat, D. (2020). Stiffness Analysis of a New Tensegrity Mechanism based on Planar Dual-triangles. In Proceedings of the 17th International Conference on Informatics in Control, Automation and Robotics Volume 1: ICINCO, ISBN 978-989-758-442-8, pages 402-411. https://doi.org/10.5220/0009803104020411

Zhao, W., Pashkevich, A., Klimchik, A., \& Chablat, D., 2020. The Stability and Stiffness Analysis of a DualTriangle Planar Rotation Mechanism. IDETCCIE2020. https://doi.org/10.1115/DETC2020-22076

Zhao, W., Pashkevich, A., Klimchik, A., \& Chablat, D., (2021). Kinematic Control of Compliant Serial Manipulators Composed of Dual-Triangles, in 2021 International Conference on Computer, Control and Robotics (ICCCR), Jan. 2021, pp. 93-97, doi: $\underline{10.1109 / I C C C R 49711.2021 .9349285}$ 